Forschung

\section{Riechrezeptoren in der Haut entdeckt}

Hautzellen besitzen einen Riechrezeptor für Sandelholzduft. Das fanden die Forscher um D. Busse von der Ruhr-Universität Bochum heraus. Ihre Daten, die im Juli im Journal of Investigative Dermatology veröffentlicht wurden, zeigen, dass sich die Teilungsrate der Zellen erhöht und Wunden besser heilen, wenn die Rezeptoren aktiviert sind. Der Mechanismus ist ein möglicher Ansatzpunkt für neue Medikamente und Kosmetika. Menschen besitzen in der Nase rund 350 verschiedene Arten von Riechrezeptoren. Auch in anderen Geweben konnte die Funktion dieser Rezeptoren nachgewiesen werden, z.B. auf Spermien, in der Prostata, dem Darm und der Niere. Das Bochumer Team entdeckte sie nun auch in Keratinozyten, den Zellen, die die äußerste Schicht der Haut bilden.

Die Wissenschaftler untersuchten den in der Haut vorkommenden Duftrezeptor OR2AT4 genauer und fanden heraus, dass ihn ein synthetischer Sandelholzduft, Sandalore genannt, aktiviert. Das Sandelholzaroma kommt häufig in Räucherstäbchen vor und ist auch eine Komponente mancher Parfüms. Der aktivierte OR2AT4Rezeptor stößt in der Zelle einen kalziumabhängigen Signalweg an. Dieser sorgt dafür, dass sich Hautzellen vermehrt teilen und schneller wandern - Prozesse, die typischerweise die Wundheilung verbessern. In Zusammenarbeit mit der Universität in Münster zeigten die Bochumer Zellphysiologen diesen Effekt an Hautzellkulturen und -explantaten.

Neben OR2AT4 haben die Wissenschaftler noch eine Reihe weiterer Duftrezeptoren in der Haut gefunden, deren Funktion sie in Zukunft genauer charakterisieren wollen. „Die Ergebnisse zeigen, dass sie therapeutisches und kosmetisches Potenzial besitzen“, so einer der Mitautoren. „Wir sollten aber auch im Hinterkopf behalten, dass man mit konzentrierten Duftstoffen vorsichtig umgehen sollte, solange wir nicht wissen, welche Funktion die verschiedenen Duftrezeptoren in Hautzellen haben.“

Nach einer Mitteilung der Ruhr-Universität Bochum

\title{
Jobwechsel verbessert berufsbedingte Symptome
}

\author{
Unter den berufsbedingten Erkrankungen nehmen bspw. in \\ Dänemark Hautkrankheiten die Spitzenposition ein. Bei mehr \\ als $90 \%$ handelt es sich um eine Kontaktdermatitis, die häufig \\ mit einer schlechten Prognose einhergeht. K. K. B. Clemmensen \\ et al. haben nun die Prognosen von Patienten, die allergisch auf \\ Gummichemikalien und Latex (ubiquitäre Allergene) bzw. \\ Epoxide (nicht ubiquitäre Allergene) reagierten, verglichen. \\ $\mathrm{Br}$ J Dermatol 2014; 170: 1100-1105
}

Die dänische Studie schloss 121 Patienten mit einer Kontaktallergie gegen Chemikalien im Gummi und 44 mit einer Epoxid-Allergie ein. Zudem wurden 27 Studienteilnehmer mit einer Kontakturtikaria aufgrund von Naturlatex sowie $7 \mathrm{~Pa}-$ tienten, die sowohl auf Gummichemikalien als auch auf Latex allergisch reagierten, untersucht. Letztere gingen allerdings nicht in die Analyse ein. Alle Fälle wurden zwischen Januar und Dezember 2010 vom Danish National Board of Industrial Injuries (DNBII) registriert. Die Wissenschaftler erfassten nach Ablauf von 2 Jahren mit einem Fragebogen den Schweregrad der Ekzeme, den Jobstatus sowie die dermatologische Lebensqualität (Dermatological Life Quality Index, DLQI; hohe Werte signalisieren eine starke Einschränkung der Lebensqualität, niedrige hingegen eine geringe Einschränkung).

\section{Kontakturtikaria mit schlechterer Prognose assoziiert}

Die Antwortrate der Studienteilnehmer lag bei $75 \%$. Das Durchschnittsalter der Patienten, die den Fragebogen ausgefüllt zurückschickten, betrug zu Studienbeginn 42,2 Jahre (Spanne: 16-65 Jahre). Während des Follow-ups verschwanden bei insgesamt $11 \%$ der Patienten die Ekzeme:

- $22 \%$ der Patienten mit einer Allergie gegen Epoxide und

- $10 \%$ mit einer Allergie gegen Gummichemikalien gegenüber

- $0 \%$ der Patienten, die unter einer Kontakturtikaria litten $(\mathrm{p}=0,116)$.
Bei 67\% der Studienteilnehmer verzeichneten die Autoren eine Verbesserung des Hautzustandes. Dies traf deutlich häufiger auf Patienten zu, die ihre Arbeitsstelle gewechselt hatten $(p=0,01)$. Die DLQIWerte waren in der Gruppe von Patienten mit Epoxid-Allergie am niedrigsten (mittlerer Wert $=1$ ) und in der Gruppe von Patienten mit Naturlatex-Allergie am höchsten (mittlerer Wert =5; $\mathrm{p}=0,016$ ).

\section{Fazit}

Innerhalb der Studie waren Patienten mit einer Kontakturtikaria im Vergleich zu Patienten, die unter einer Kontaktallergie gegen Gummichemikalien litten, durch eine deutlich schlechtere Prognose gekennzeichnet. Bei einem Vergleich zwischen Patienten mit einer Kontaktallergie gegen Chemikalien im Gummi und solchen mit einer Epoxid-Allergie wiesen Erstere eine tendenziell schlechtere Prognose auf. Die Autoren identifizierten zudem eine positive Assoziation zwischen einem Jobwechsel und einer Verbesserung des Hautzustandes.

\section{Dr. Frank Lichert, Weilburg}

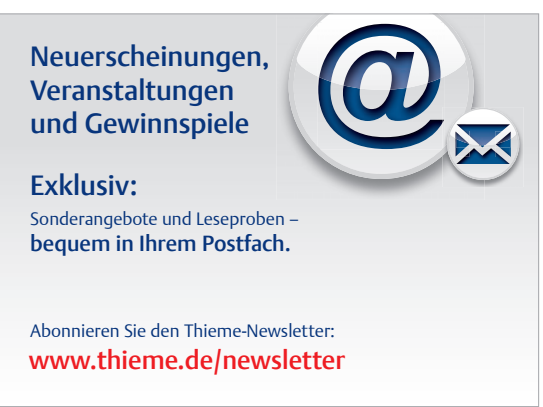

